\title{
Cancer Immunotherapy Comes of Age
}

Suzanne L. Topalian, George J. Weiner, and Drew M. Pardoll

Suzanne L. Topalian and Drew M. Pardoll, Johns Hopkins University School of Medicine and Sidney Kimme Comprehensive Cancer Center, Baltimore, MD; and George J. Weiner, Holden Comprehensive Cancer Center, University of lowa, lowa City, IA

Submitted July 5, 2011; accepted September 23, 2011; published online ahead of print at www.jco.org on October 31, 2011.

Supported by National Institutes of Health (NIH) Grants No. P30 CA86862, P50 CA97274, and R01 CA137198 (G.J.W.); NIH Grant No. R01 CA142779 (S.L.T., D.M.P.); the Melanoma Research Alliance (S.L.T., D.M.P.); and the Barney Family Foundation (S.L.T.).

Terms in blue are defined in the glossary, found at the end of this article and online at www.jco.org.

Authors' disclosures of potential conflicts of interest and author contributions are found at the end of this article.

Corresponding author: Suzanne L. Topalian, MD, 1550 Orleans St, David H. Koch Cancer Research Building, Room 508, Baltimore, MD; e-mail: stopali1@jhmi.edu.

(C) 2011 by American Society of Clinica Oncology

$0732-183 \times / 11 / 2936-4828 / \$ 20.00$ DOI: 10.1200/JCO.2011.38.0899

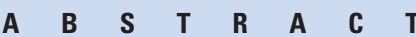

Cancer immunotherapy comprises a variety of treatment approaches, incorporating the tremendous specificity of the adaptive immune system (T cells and antibodies) as well as the diverse and potent cytotoxic weaponry of both adaptive and innate immunity. Immunotherapy strategies include antitumor monoclonal antibodies, cancer vaccines, adoptive transfer of ex vivo activated T and natural killer cells, and administration of antibodies or recombinant proteins that either costimulate immune cells or block immune inhibitory pathways (so-called immune checkpoints). Although clear clinical efficacy has been demonstrated with antitumor antibodies since the late 1990s, other immunotherapies had not been shown to be effective until recently, when a spate of successes established the broad potential of this therapeutic modality. These successes are based on fundamental scientific advances demonstrating the toleragenic nature of cancer and the pivotal role of the tumor immune microenvironment in suppressing antitumor immunity. New therapies based on a sophisticated knowledge of immune-suppressive cells, soluble factors, and signaling pathways are designed to break tolerance and reactivate antitumor immunity to induce potent, long-lasting responses. Preclinical models indicate the importance of a complex integrated immune response in eliminating established tumors and validate the exploration of combinatorial treatment regimens, which are anticipated to be far more effective than monotherapies. Unlike conventional cancer therapies, most immunotherapies are active and dynamic, capable of inducing immune memory to propagate a successful rebalancing of the equilibrium between tumor and host.

\section{J Clin Oncol 29:4828-4836. (C) 2011 by American Society of Clinical Oncology}

\section{INTRODUCTION}

The relationship between the immune system and human cancer is dynamic and complex. Individual human tumors harbor a multitude of somatic gene mutations and epigenetically dysregulated genes, the products of which are potentially recognizable as foreign antigens. ${ }^{1}$ However, the overriding relationship between the immune system and growing cancers is one of tolerance, in which, paradoxically, foreign molecules expressed by tumor cells are viewed as self. ${ }^{2}$

Growing cancers contain tumor-infiltrating lymphocytes (TILs), which are ineffective at tumor elimination in vivo but can exert specific functions (eg, proliferation, cytokine secretion, cytolysis) outside the immunosuppressive and toleragenic tumor microenvironment. This is because the tumor milieu contains suppressive elements including regulatory $\mathrm{T}$ cells and myeloid-derived suppressor cells; soluble factors such as interleukin 6 (IL-6), IL-10, vascular endothelial growth factor, and transforming growth factor beta; and ligands for coinhibitory receptors that downmodulate TIL activity. ${ }^{3}$ The clinical responsiveness of melanoma and renal cell carcinoma (RCC) to systemically administered proinflammatory cytokines such as IL-2 demonstrates the antitumor potential of an activated immune system; however, this nonphysiologic method of reversing immunologic tolerance exerts global rather than localized effects, resulting in serious systemic toxicities. $^{4,5}$ The recent molecular characterization of toleragenic mechanisms mediated by human tumors has sharpened the focus of cancer immunotherapy on more specifically targeted methods for overcoming tolerance, revealing new therapeutic opportunities. Promising immunotherapies based on recombinant and cellular agents that harness innate as well as adaptive immune responses are the subject of this review. They illustrate the diversity of antitumor immunity and highlight the need to incorporate multiple approaches into synergistic combinatorial treatment strategies.

TUMOR-SPECIFIC MONOCLONAL ANTIBODIES

Monoclonal antibodies (mAbs) have had a major impact on the practice of clinical oncology. Indeed, 
the three top-selling cancer drugs (ie, rituximab, trastuzumab, and bevacizumab) are mAbs. Most preclinical models used to test mAbs are not designed to assess the active role of the host immune response in mediating mAb-induced anticancer responses, resulting in an underestimation of the importance of this phenomenon. In vitro assays exploring the immune effects of anticancer mAbs involve extensively manipulated lymphocytes and span a few hours. In contrast, therapeutic levels of mAbs are present for months in treated patients, allowing for more extensive lymphocyte trafficking and activation and lysis of cancer cells. In vivo studies of mAbs often involve animal tumor models with limited heterogeneity, extremely rapid growth, and limited infiltration with immune effector cells; many are performed with xenografts in immunodeficient mice.

Despite these limitations, data supporting the role of the immune response in general, and antibody-dependent cell-mediated cytotoxicity (ADCC) in particular, as a major mechanism of mAb activity are convincing. These data are strongest for rituximab. ${ }^{6}$ Studies in vitro, animal models, and correlative clinical investigations indicate that the interaction between $\mathrm{mAb}$ and $\mathrm{Fc}$ receptor $(\mathrm{FcR})$ contributes to the clinical antitumor activity of rituximab. Patients with lymphoma and a polymorphism encoding high-affinity FcR (more specifically, Fc $\gamma$ RIII) have a better response rate to single-agent rituximab than do patients with low-affinity FcR. ${ }^{7-9}$ Cancers growing in mice lacking activating FcR fail to respond to anticancer mAbs, including rituximab and trastuzumab. ${ }^{10}$ Trastuzumab can alter human epidermal growth factor receptor 2 signaling; its ability to mediate ADCC likely also contributes significantly to its antitumor activity. ${ }^{11}$ This also applies to other mAbs that target antigens on the surface of cancer cells such as other epidermal growth factor receptor family members.

There is growing evidence of extensive interactions-both synergistic and antagonistic - among various mechanisms of action that modulate the immune effects of mAbs (Fig 1). mAb-induced tumor cell lysis mediated by a number of different mechanisms may lead to enhanced uptake and cross presentation of the targeted antigen, thereby generating an adaptive cellular anticancer immune response, at least in preclinical models. ${ }^{12}$ Complement has complex effects. There is little evidence that complement-mediated cytotoxicity (CMC) contributes to antitumor activity in solid tumors, but it may contribute in hematologic malignancies, in which target cells are exposed to complement in the circulation. ${ }^{13}$ The anti-CD20 mAbs rituximab and ofatumumab, and the anti-CD52 mAb alemtuzumab, kill target cells rapidly in vitro via CMC. On the other hand, complement can block interactions between $\mathrm{mAb}$ and activating $\mathrm{FcR}$ on natural killer (NK) cells and may reduce ADCC. Components of complement clear apoptotic bodies, thereby limiting development of an active immune response. ${ }^{14}$ Thus, in some circumstances, complement may mediate CMC and enhance response to $\mathrm{mAb}$, whereas in others, it could inhibit ADCC-dependant anticancer immune responses and so blunt responses to $\mathrm{mAb}$.

The majority of mAbs approved for clinical use contain a human immunoglobulin (Ig) G1 heavy chain. For many years, investigators have tried to enhance the effects of mAb therapy by producing modified mAbs and conjugates. Persistence has paid off, and these approaches are finally showing promise. Changing the sequence or glycosylation of Fc regions can enhance interactions with FcR on immune effector cells. ${ }^{15,16}$ Bifunctional antibody-like molecules that bind to an antigen on a cancer cell with one arm and activating molecules such as CD3 with the other are being used to retarget T cells toward cancer cells. ${ }^{17,18}$ Drug-antibody conjugates are also showing considerable promise, particularly in hematologic malignancies. ${ }^{19,20}$ Although much of the antitumor effect of such conjugates results from the cytotoxic effects of the drugs, it is likely that immune response also plays a role.

Moving forward, how can knowledge about the immune effects of anticancer mAbs influence our ability to use them therapeutically? First, such information informs the identification of biomarkers that

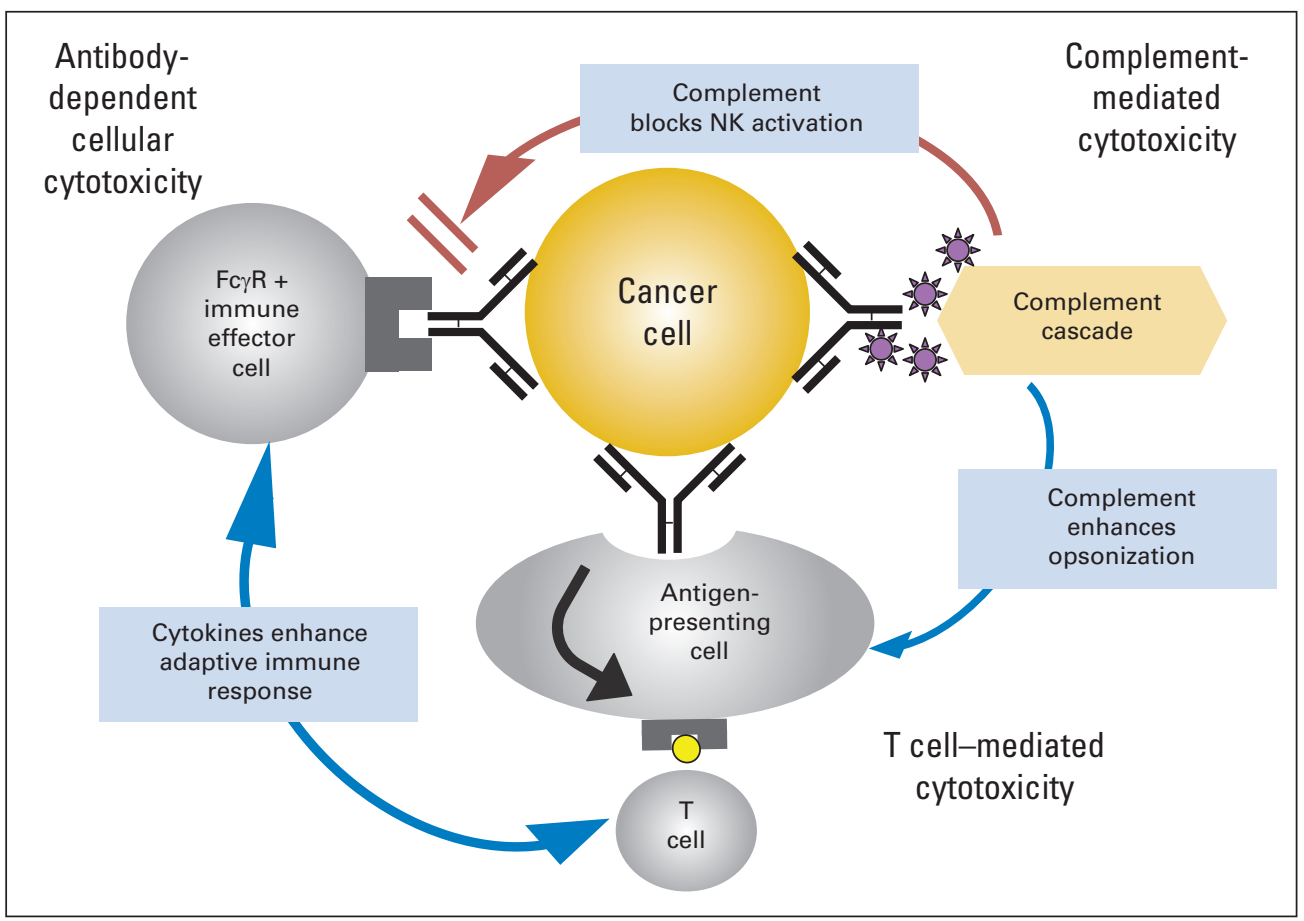

Fig 1. Multiple immunologic mechanisms contribute to anticancer effects of monoclonal antibodies, including antibody-dependent cellular cytotoxicity, complement-mediated cytotoxicity, and enhancement of the adaptive immune response. These mechanisms do not function in isolation, with importance of individual mechanism varying based on type of cancer, monoclonal antibody used, patient characteristics including host genetic polymorphisms, and location of cancer. Fc $\gamma R$, Fc $\gamma$ receptor; NK, natural killer. 
can enhance our ability to select those patients who are most likely to respond to $\mathrm{mAb}$ therapy. The most obvious biomarker is tumor expression of the target antigen. Genetic polymorphisms, including polymorphisms in Fc $\gamma \mathrm{R}$ and complement, can also affect the likelihood of response to mAb therapy. Second, information on mechanisms of action supports the development of combinatorial therapies of mAbs with other forms of cancer therapy, including agents that activate NK cells or other immune effector cells. Finally, we now have the ability to modify mAb structure and thereby modulate effector mechanisms such as the ability to bind to FcR or fix complement. Understanding the mechanisms of action responsible for the immune effects of antitumor mAbs, and using this information to apply and modify mAbs in a way that enhances these effects, will allow us to optimize this already effective class of immunotherapeutic agents.

\section{ADOPTIVE CELL TRANSFER}

Adoptive cell transfer (ACT) is a form of immunotherapy in which antitumor $\mathrm{T}$ cells are manipulated ex vivo and then infused into the patient. The earliest form of effective ACT was bone marrow transplantation (BMT) for hematologic malignancies. Enhanced graft versus leukemia/lymphoma effects from allogeneic versus autologous and T cell-replete versus depleted BMT were associated with improved clinical outcomes, although at the cost of increased graft versus host disease. $^{21,22}$ In patients with relapsing chronic myeloid leukemia or indolent lymphoma after allogeneic BMT, the efficacy of donor lymphocyte infusions directly demonstrated the antitumor activity of transferred lymphocytes. ${ }^{23,24}$ Although T cells were long presumed to be responsible for graft versus leukemia/lymphoma effects, recent studies have elucidated an important role for alloreactive NK cells when donor killer inhibitory receptors are incompatible with host ligands. ${ }^{25}$

With the discovery in the 1980s that human T cells isolated from peripheral blood, tumor-draining lymph nodes, or tumor tissue could manifest selective antitumor reactivity in vitro, the cancer immunotherapy field undertook to develop specifically targeted ACT protocols. Melanoma TILs are a rich source of tumor-specific CD4+ and CD8 + T cells relative to other malignancies. ${ }^{26}$ The heightened immunogenicity of melanoma compared with other human cancers has provided a model system in which to define and characterize tumor antigens, comprising shared nonmutated tumor-associated proteins as well as uniquely mutant molecules. ${ }^{27}$ Autologous unfractionated TILs expanded in vitro and infused into patients with metastatic melanoma, in conjunction with systemic IL-2, have mediated objective responses in $34 \%$ to $50 \%$ of patients. ${ }^{28,29}$ Biomarker studies correlating clinical responses with the in vitro tumor specificity of $\mathrm{TIL}^{30}$ have led to the development of more complex methods to subculture tumor-reactive cells. Combined with more intense chemoradiotherapy preconditioning regimens, objective clinical response rates of $49 \%$ to $72 \%$ were observed in patients with melanoma receiving highly selected TILs. ${ }^{31}$ Because clinical TIL studies have been performed sequentially and have not been randomized, these different treatment regimens cannot be directly compared.

In contrast to the substantial therapeutic impact of ACT with polyclonal TIL cultures, reduced clinical efficacy has been encountered with $\mathrm{CD} 4+$ or CD8 + clones specific for a single melanoma antigen (MART-1/Melan-A, gp100, NY-ESO-1; Fig 2). ${ }^{32-34}$ Clinical

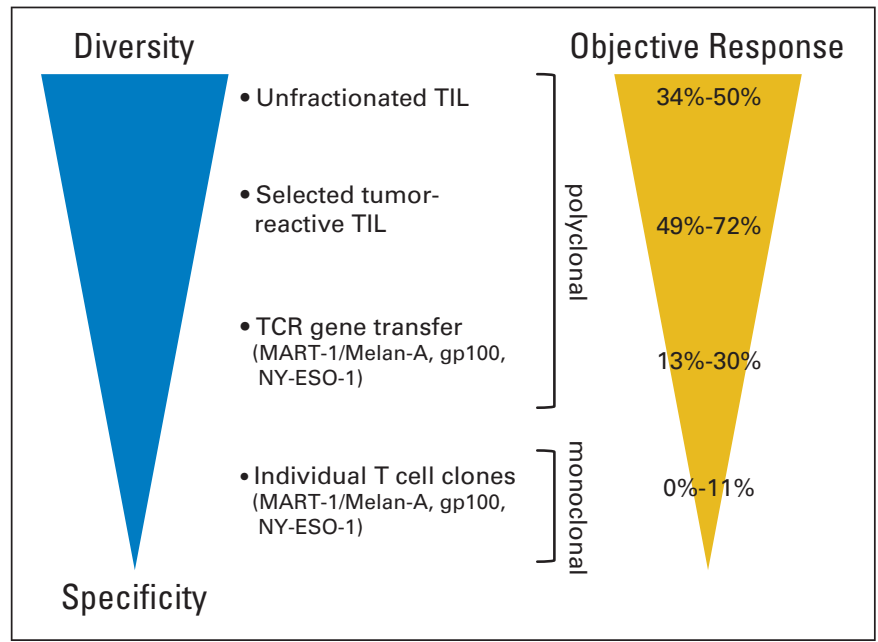

Fig 2. Association between immunologic diversity of transferred $T$ cells and improved clinical outcomes from adoptive cell transfer (ACT) in patients with metastatic melanoma. Autologous unfractionated tumor-infiltrating lymphocytes (TILs) infused in conjunction with systemic interleukin 2 yielded objective responses in $34 \%$ to $50 \%$ of patients. ${ }^{28,29}$ Biomarker studies correlating clinical responses with in vitro TIL properties of tumor-specific cytolysis and cytokine secretion led to development of more complex culture methods to deliberately select tumor-reactive subcultures for therapy. Combined with more intense chemoradiotherapy preconditioning regimens, objective clinical response rates of $49 \%$ to $72 \%$ were achieved with selected TILs. ${ }^{31}$ In contrast, lower response rates were observed in $A C T$ studies using $T$-cell receptor (TCR) -transduced T cells (mixtures of CD4+ and CD8+ cells) ${ }^{35}$ or monoclonal CD4+ or CD8+ T-cell cultures specific for single melanoma antigen (MART-1/Melan-A, gp100, NYESO-1). ${ }^{32-34}$ Outgrowth of antigen-loss tumor variants in these patients, reflecting successful antigen targeting, also indicated capacity of rapidly adaptable tumor cells to evade narrowly focused therapies. Although these summarized results are gleaned from nonrandomized ACT studies, there seems to be association between immunologic diversity of infused cells and likelihood of clinical activity.

trials with T-cell clones have provided a platform for proof-of-principle studies, including precise monitoring of proliferation, trafficking, and persistence of transferred cells. The outgrowth of antigen-loss tumor variants in treated patients has reflected successful antigen targeting while underscoring the capacity of rapidly adaptable tumor cells to evade narrowly focused therapies. Reduced persistence and/or trafficking of T-cell clones may also underlie reduced clinical activity.

Individualized and cumbersome microculture techniques for cloning tumor-reactive $\mathrm{T}$ cells are being superseded by genetically engineered T-cell receptor (TCR) -transduced T cells for ACT, in which genes encoding TCRs with defined antitumor properties are transduced into short-term cultured peripheral blood lymphocytes $(\mathrm{CD} 4+$ and $\mathrm{CD} 8+)$, conferring tumor recognition. This therapy is potentially accessible to any patient whose tumor possesses the cognate human leukocyte antigen (HLA) allele and expresses the target antigen recognized by the TCR. Objective response rates after ACT with HLA-A2-restricted TCRs against MART-1/Melan-A or gp100 have ranged from $13 \%$ to $19 \%$ using native TCRs to $30 \%$ with modified TCRs designed to enhance normally low TCR avidities for tumor major histocompatibility complex (MHC) -antigen complexes. ${ }^{35}$ However, the clinical use of highly avid TCRs has been associated with significant collateral destruction of normal tissues sharing the target antigen. Current efforts aim to optimize gene transfer efficiencies, design TCR structural modifications, and identify target antigens, the expression of which is highly selective in tumor rather than nontransformed cells. ${ }^{36}$ ACT with an HLA-A2-restricted TCR specific for 
NY-ESO-1, a cancer-testis antigen expressed by a variety of human cancers and testis but not other normal adult tissues, resulted in objective tumor regressions in five of 11 patients with melanoma without incurring serious autoimmune toxicities. In the same study, four of six patients with treatment-refractory synovial cell sarcoma demonstrated objective responses, ${ }^{37}$ showing that principles of immunotherapy established in melanoma can now be successfully extended to other forms of cancer.

Chimeric antigen receptors (CARs) were developed to overcome limitations of $\mathrm{MHC}$ restriction and intracellular antigen processing imposed by ACT with conventional T cells. CARs are single-chain constructs composed of an Ig variable domain (extracellular) fused to a TCR constant domain (extracellular/intracellular); when introduced into T cells, they combine the antigen-recognition properties of antibodies with T-cell lytic functions, broadening the spectrum of tumor antigen recognition. ${ }^{38}$ To optimize CAR signaling and activation potential, first-generation CARs incorporating the cytoplasmic domain of CD3-zeta were followed by second- and third-generation CARs, the cytoplasmic domains of which incorporate multiple signaling modules from costimulatory receptors. ${ }^{39}$ Encouraging early clinical results with second-generation anti-CD19 CARs have been observed in patients with lymphoma. ${ }^{40,41}$ However, the high affinity for target cells conferred by the Ig component of CARs, combined with amplified nonphysiologic T-cell signaling in second- and third-generation constructs, has been associated with serious adverse events. ${ }^{42}$ Reducing on-target toxicities while maintaining antitumor efficacy is an important goal of current investigations. Possible solutions include use of lower-affinity Ig variable domains, more judicious selection of target antigens, infusion of defined $\mathrm{T}$ cell subsets, methodic dose escalation, alternative gene transfer technologies, ${ }^{43}$ and co-opting of the more physiologic T-cell signaling mechanisms of virus-specific T cells. ${ }^{44}$

\section{CANCER VACCINES}

Historically, the primary approach to specifically activate host $\mathrm{T}$ cells against tumor antigens (ie, active immunotherapy) has been therapeutic cancer vaccination. Long-standing interest in cancer vaccines comes from the tremendous successes of prophylactic vaccines for infectious diseases and is based on immunobiology demonstrating the capacity of $\mathrm{T}$ cells to recognize target antigens in the form of peptides complexed to surface MHC molecules. Because immunogenic peptides can be derived from proteins in every cellular compartment, essentially any protein has the potential to be recognized by T cells as a tumor-specific or tumor-selective antigen. Successful vaccination marshals multiple immune effector arms including CD4+ and CD8+ T cells to generate a potent antitumor response. ${ }^{45}$

Despite anecdotal reports and promising phase I and II clinical trial results with cancer vaccines evaluated since the 1960s, a string of failures in randomized clinical trials has bred significant skepticism as to the ultimate clinical value of therapeutic cancer vaccines. ${ }^{46-48}$ However, in the past few years, a number of important successes with cancer vaccines have dramatically altered the perception of their potential value.

The first successful randomized phase III cancer vaccine trial used a putative dendritic cell (DC) vaccine-sipuleucel-T-to treat patients with advanced hormone-resistant prostate cancer. ${ }^{49}$ This vaccine is based on the concept that optimal T-cell activation requires antigen processing and presentation by a specialized cell- the DCwith the capacity to concomitantly deliver strong costimulatory signals in the form of membrane ligands and secreted cytokines. Sipileucel-T is a patient-specific vaccine produced by transiently incubating the patient's own peripheral blood mononuclear cells with a fusion protein consisting of prostatic acid phosphatase (prostate/prostate cancer-specific antigen) linked to the DC growth and differentiation factor granulocyte macrophage colony-stimulating factor (GMCSF). A 4-month overall survival (OS) benefit relative to the control arm (uncultured peripheral blood mononuclear cells without prostatic acid phosphatase-GM-CSF fusion protein) in the absence of objective tumor regressions or effect on time to progression emphasizes a developing paradigm: immunotherapy can potentially provide OS benefits that are not reflected in progression-free survival (PFS) or objective response rate (ORR). The survival benefit of sipuleucel-T ultimately led to US Food and Drug Administration approval in 2010.

Recently, two positive randomized cancer vaccine trials were reported. A melanoma vaccine consisting of a modified gp100 peptide plus systemic IL-2 was compared with systemic IL-2 alone in patients with advanced melanoma, ${ }^{50}$ yielding a statistically higher ORR in the vaccine plus IL-2 arm, improved PFS, and improved OS $(P=.06)$. Of note, the same peptide vaccine, when combined with anti-cytotoxic T-lymphocyte antigen 4 (CTLA-4), demonstrated no improvement in patients with advanced melanomas relative to anti-CTLA-4 alone, ${ }^{51}$ underscoring the importance of context when evaluating vaccines as components of combinatorial therapies. Another trial comparing a poxvirus-prostate specific antigen prime/boost vaccine regimen plus GM-CSF versus nonantigen expressing viruses in patients with advanced prostate cancer demonstrated a significant (8 months) OS benefit for the vaccine arm but no effect on PFS or ORR. ${ }^{52}$ Finally, a single-arm clinical trial using long peptides (selectively processed and presented by DCs) derived from human papillomavirus 16 (HPV-16) E6 and E7 antigens induced complete regressions in nine of 19 patients with vulvar intraepithelial neoplasia, an HPV-associated preneoplastic condition, with a spontaneous regression rate of less than $2 \% .{ }^{53}$

Why has there been a recent spate of successful cancer vaccine trials after such a long drought? Basic immunology advances in the past decade have directly affected vaccine design and clinical trial design. We now know that as cancer grows, it induces tolerance among $\mathrm{T}$ cells specific for its antigens, ${ }^{54}$ usurping normal mechanisms of self tolerance and dampening immune responses within the microenvironment through a variety of mechanisms. ${ }^{55,56}$ Thus, in contrast to conventional prophylactic vaccines for infectious diseases, therapeutic cancer vaccines must break tolerance to reactivate antitumor immune cells. Critical means for achieving this goal include targeting high quantities of antigen to DCs, expanding DC numbers, and providing DCs with appropriate activation signals (Fig 3). ${ }^{57}$ Activated DCs traffic to draining lymph nodes, where they present antigen to T cells. Although locally elaborated GM-CSF dramatically expands DC numbers at the vaccine site, additional signals are required for DC activation. These are mediated by pattern-recognition receptors (PRRs; toll-like receptors), which bind common microbial molecules, and a set of cytosolic sensors (eg, melanoma differentiation-associated antigens, retinoic acid-inducible gene 1 , and nucleotide-binding oligomerization domain-leucine-rich repeat proteins). ${ }^{58}$ Most earlygeneration cancer vaccines did not incorporate agonists for these PRRs and thus would not have been expected to break tolerance; 


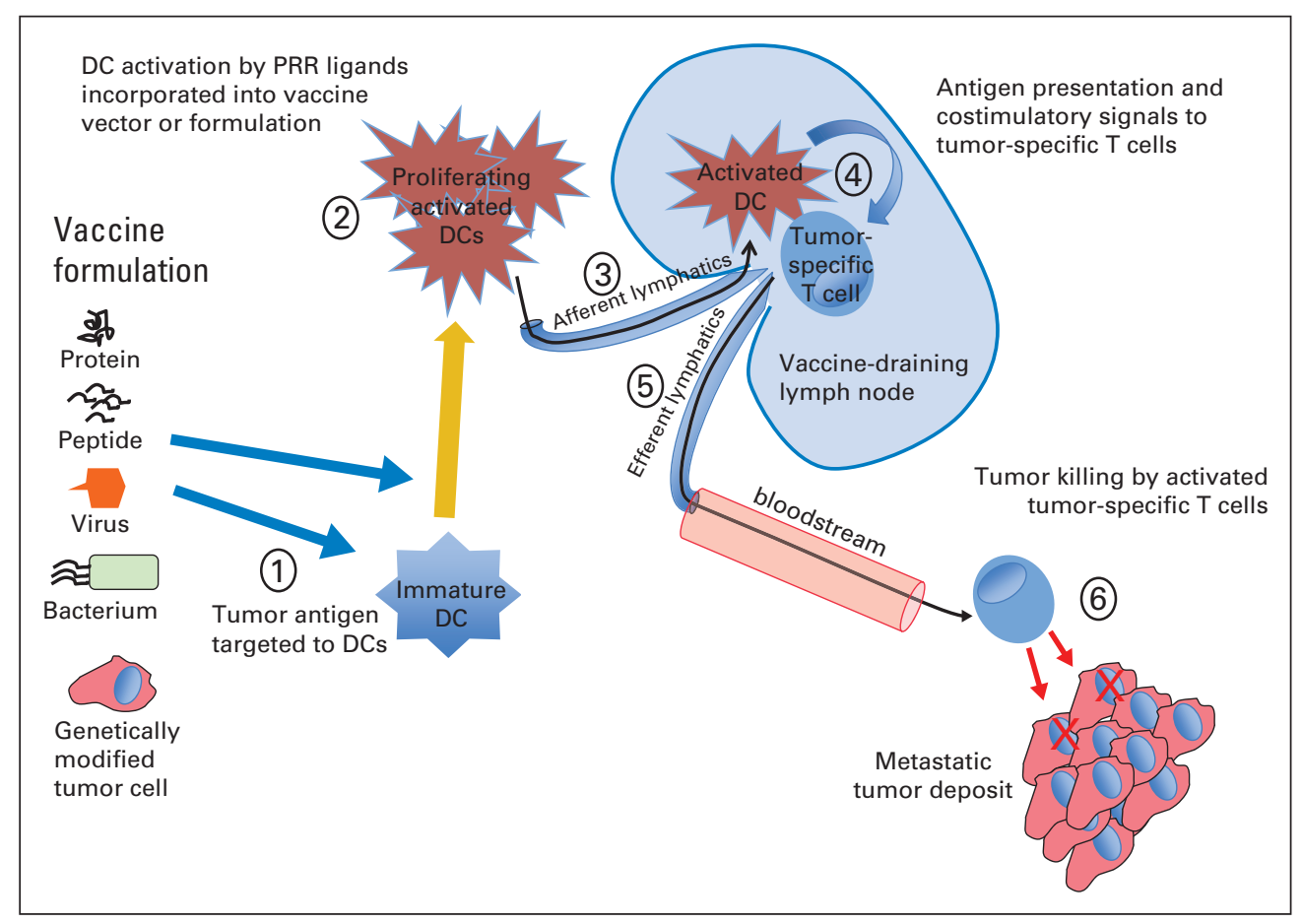

Fig 3. Critical role of dendritic cells (DCs) in generating vaccine-induced antitumor immune responses. Cancer vaccines consist of many diverse formulations, in which antigen is in form of protein or peptide, recombinant virus or bacterium, or engineered tumor cell. Ultimately, antigen must be targeted to DCs (1). For cancer vaccine to be effective, DCs must be activated, either through incorporation of pattern-recognition receptor (PRR) agonist into vaccine or via activation properties of vector (ie, virus or bacterium). Ideal viral or bacterial vaccine vectors can infect DCs and, in so doing, activate them (2). Steps 1 and 2 can be accomplished ex vivo, as with DC vaccines (ie, sipuleucel-T). Activated DCs loaded with tumor antigen traffic to draining lymph node via afferent lymphatics (3). In lymph node, they present processed antigen to $T$ cells along with costimulatory signals in form of cytokines and membrane ligands, thereby activating tumor-specific $T$ cells (4) that are otherwise in tolerant state. Activated $T$ cells leave draining lymph nodes via efferent lymphatics (5) and ultimately enter bloodstream via thoracic duct. They exit bloodstream in peripheral tissues, where they seek out and recognize tumor deposits expressing cognate tumor antigen and exert antitumor effects (6).

however, newer vaccine formulations are indeed incorporating both synthetic and natural PRR ligands.

Another component of vaccines in which scientific advances can be leveraged is the choice of target antigen. Many earlier vaccine formulations used whole cells or cell lysates as polyvalent sources of tumor antigens because relevant tumor-specific or tumor-selective antigens had not yet been defined. However, cell-based vaccines contain thousands of self antigens that provide no tumor specificity. For $\mathrm{T}$ cells, specific tumor antigens were first defined by the pioneering work of Boon et $\mathrm{al}^{59}$ and Kawakami et $\mathrm{al}^{60}$ in melanoma. Since then, tumor antigens recognized by $\mathrm{T}$ cells from patients with cancer have been defined for many common cancer types. The ideal tumor antigen is: first, expressed in a significant proportion of patients with a particular cancer type; second, not expressed (or expressed at low levels) in normal tissues; and third, vital to the cancer's growth and/or survival (avoiding outgrowth of resistant antigen-loss tumor variants). Relatively few antigens in current cancer vaccines fit all these criteria, although viral oncogene products in virus-associated cancers (ie, HPV E6 and E7 ${ }^{61}$ ) as well as certain self antigens, such as Wilms tumor 1 (leukemia ${ }^{62}$ ) and mesothelin (pancreas, ovarian, and lung cancers ${ }^{63}$ ) do. Thus, these findings have enabled the development of antigen-specific vaccines that can be engineered to codeliver tumor antigens with DC activation signals, optimally promoting effective antitumor immunity.

\section{IMMUNE-MODULATING ANTIBODIES}

In addition to shared antigens selectively expressed by tumors, investigations into the nature of the human antitumor immune response have revealed a vast array of unique antigenic targets derived from mutated genes found in individual tumors. These are not approachable with generic methods of immunization, ACT, or tumor-specific
mAbs. With the relatively recent realization that cancer exerts an immune-tolerizing influence in the host, new trends in immunotherapy have focused on methods to interrupt tolerogenic pathways and reactivate endogenous immunity against unique as well as shared tumor antigens.

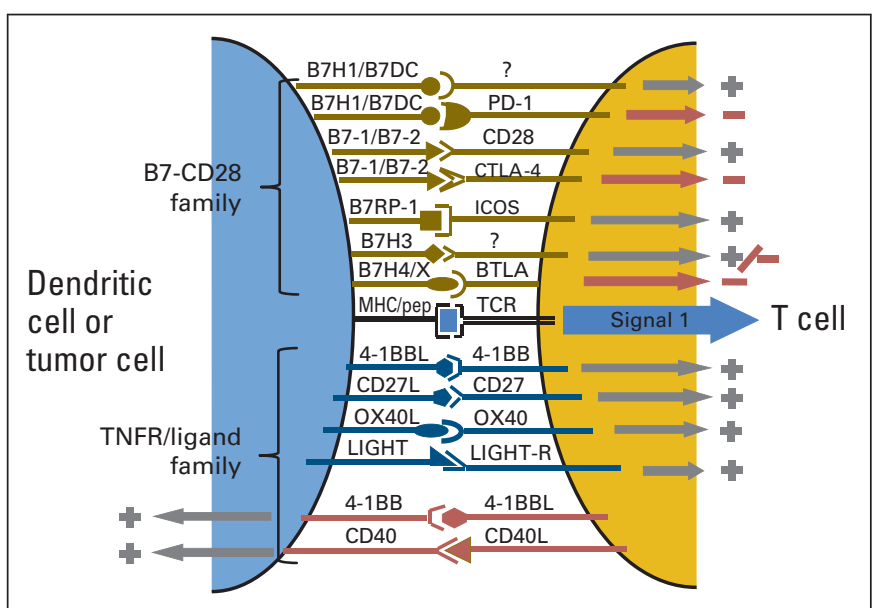

Fig 4. Immunologic synapse. Target recognition by $T$ cells is two-step process. Specific interaction of T-cell receptor (TCR) with major histocompatibility complex ( $\mathrm{MHC}$ - -peptide complexes displayed by tumor cells or antigen-presenting cells (APCs; eg, dendritic cells) provides first signal for T-cell recognition. Second event is coregulatory signal that determines whether $T$ cell will become activated or anergic (nonreactive). T-cell coreceptors transmitting stimulatory $(+)$ or inhibitory $(-)$ signals on engagement of specific ligands expressed by tumor cells or APCs are depicted. Molecules in B7-CD28 and tumor necrosis factor receptor (TNFR) families are now being targeted for cancer immunotherapy. 4-1BBL, 4-1BB ligand; BTLA, B- and T-lymphocyte attenuator; CTLA-4, cytotoxic T-lymphocyte antigen 4; ICOS, inducible T-cell costimulator; LIGHT, homologous to lymphotoxin, shows inducible expression, competes with herpes simplex virus glycoprotein D, expressed by T cells; PD-1, programmed cell death 1 . 
The fine specificity of T cells for their targets is mediated by the interaction of TCRs with antigenic peptide-MHC complexes displayed on the cell surface. However, the functional consequences of antigen recognition are mediated by coregulatory receptors expressed on T cells, which recognize cognate ligands displayed on target cells including antigen-presenting cells and tumor cells (Fig 4). These coreceptors can induce stimulatory or inhibitory signaling cascades, thereby modulating T-cell proliferation, cytokine secretion, and cytolysis. A dominance of coinhibitory receptor ligation induces tolerance. The best-studied group of coregulatory molecules is the CD28-B7 family, ${ }^{64}$ and a receptor for B7-1 and B7-2 termed CTLA- 4 was the initial target for immune-modulatory antibodies. CTLA-4 is a coinhibitory TCR, the natural function of which is to downmodulate immunity at the appropriate time, avoiding collateral normal tissue damage. Although there is no tumor specificity in the expression of B7-1 or B7-2, potent antitumor properties of CTLA-4 blocking mAbs were nonetheless observed in preclinical models ${ }^{65}$ and then validated in the clinic. Two anti-CTLA-4 blocking mAbs-ipilimumab (Bristol-Myers Squibb, Princteon, NJ) and tremelimumab (Pfizer, New York, NY) - demonstrated similar properties in early-phase clinical trials in patients with advanced solid tumors, mediating objective response rates of $10 \%$ to $15 \%$ in patients with metastatic melanoma and RCC. ${ }^{66-68}$ Response characteristics included delayed onset, mixed regressions (ie, concomitant regressing/progressing lesions), and long-term complete remissions in a small percentage of patients. Ipilimumab (Yervoy; Bristol-Myers Squibb) was recently approved as first-line therapy for patients with melanoma with metastatic disease, based on phase III trials in which this drug, administered alone or in combination with a gp100 peptide vaccine or with dacarbazine, demonstrated superior OS and PFS compared with vaccine alone $e^{51}$ or dacarbazine alone, ${ }^{69}$ respectively. Approximately $20 \%$ of patients in both studies achieved long-term survival benefit; this exceeded the reported ORRs of $10 \%$ to $15 \%$, suggesting that, as with other immunotherapies, ipilimumab may induce a state of equilibrium between the immune system and cancer, resulting in prolonged disease stabilization but not regression in some patients. As forecast by the lethal hyperimmune/autoimmune phenotype of CTLA- 4 knockout mice, ${ }^{70}$ grades 3 to 5 immune-related adverse events have been observed in $10 \%$ to $35 \%$ of patients undergoing CTLA- 4 blockade. The most frequently affected organs are colon, endocrine glands, and skin; the diverse spectrum of inflammation was unanticipated from patient medical histories.

The occurrence of immune-related adverse events validates the immunologic mechanism of action of CTLA-4 mAbs, but it also mandates the exploration of alternative immune checkpoint pathways with potentially improved benefit-to-toxicity ratios as targets for cancer therapy. Among mAbs targeting other members of the CD28-B7 family, anti-programmed cell death 1 (PD-1) and anti-B7-H1/PD ligand 1 (PD-L1) are farthest along in clinical development. This pathway is of particular interest because B7-H1/PD-L1, unlike B7$1 /-2$, is selectively upregulated by many human cancers. ${ }^{71}$ The PD-1 pathway normally plays a protective role in modulating immunemediated tissue destruction but can be exploited by cancer to protect itself from tumor-specific T cells. ${ }^{56}$ Although CTLA-4 regulates de novo immune responses, the PD-1 pathway exerts its major influence on ongoing (effector) immune responses; this is supported by the distinct phenotypes of PD-1 genetic knockout mice, which develop delayed-onset organ-specific inflammation as opposed to the uncon- trolled global T-cell proliferation seen in CTLA-4 knockouts. Of three anti-PD-1 mAbs currently in the clinic for cancer therapy-MDX1106/BMS936558 (Medarex, Princeton, NJ; Bristol-Myers Squibb), CT-011 (CureTech, Yavne, Israel), and MK-3475 (Merck, Whitehouse Station, NJ)—most experience has involved MDX-1106. ${ }^{72} \mathrm{~A}$ first-in-human phase I trial of intermittent dosing showed durable objective responses in three of 39 patients with treatment-refractory metastatic solid tumors (melanoma, RCC, and colorectal cancer), and clinical responses correlated with pretreatment expression of $\mathrm{B} 7-\mathrm{H} 1 /$ PD-L1 in the tumor. ${ }^{73}$ An ongoing trial administering MDX-1106 biweekly has shown preliminary evidence of durable objective tumor responses in approximately one third of patients with advanced melanoma and RCC; grade 3 or greater adverse clinical events occurred in $12 \%$ of 126 patients and included the same kinds of immune-related phenomena encountered with anti-CTLA- $4 .^{74,75}$ Of interest, objective tumor responses to MDX-1106 have also occurred in patients with treatment-refractory non-small-cell lung cancer, highlighting activity against a nonimmunogenic tumor. A blocking antibody against the major ligand for PD-1-B7-H1/PD-L1 (MDX-1105/ BMS936559) - is also in phase I clinical testing in patients with advanced solid tumors, and preliminary evidence of clinical activity against melanoma, RCC, and non-small-cell lung cancer has been shown. Although these results validate the PD-1 pathway as a target for immunotherapy, anti-B7-H1/PD-L1 might be expected to have a unique spectrum of clinical activity based on B7-H1/PD-L1 biology.

Momentum gained from clinical results with mAbs blocking coinhibitory pathways has generated exploratory studies of agonistic or antagonistic mAbs against new targets, including T-cell costimulatory receptors in the tumor necrosis factor receptor family. Agonistic antibodies against 4-1BB (CD137), ${ }^{76}$ OX40 (CD134), ${ }^{77}$ glucocorticoid-induced tumor necrosis factor receptor family-related gene (GITR), and CD27 are currently or soon to be in the clinic. Although these agents may be effective as monotherapies, preclinical models indicate that maximum impact will be achieved in treatment combinations exploiting their unique roles in generating and maintaining antitumor immunity.

\section{FUTURE DIRECTIONS: OPPORTUNITIES FOR} COMBINATORIAL THERAPIES

The complexity of successful immune responses with coordinate recruitment of innate and adaptive immunity, including soluble and cellular factors, has been established in preclinical and clinical models of infectious diseases and transplantation and applies to antitumor immunity as well. This review of immunotherapeutic agents in cancer barely scratches the surface of potential potency achievable by combinatorial strategies targeting distinct effector arms and both early (activation) and late (execution) stages of immune response. For example, combinations of vaccines with blocking mAbs against immune checkpoint receptors such as CTLA-4 and PD-1 demonstrate dramatic synergy in murine tumor models, in which the individual components offer little or no therapeutic efficacy. ${ }^{78,79}$ Another therapeutic opportunity involves the rational combination of distinct checkpoint inhibitors based on their biologic properties. For example, the CTLA-4 checkpoint plays a major role in dampening initial T-cell activation, whereas PD-1 inhibits effector T-cell responses within tissues. Thus, anti-CTLA-4 and anti-PD-1 have demonstrated synergy in animal tumor models, and this combination is in clinical testing. ${ }^{80}$ 
Although such combinations may significantly enhance antitumor immunity, they may also generate additive or synergistic immune toxicities, requiring careful dose titrations to define windows of clinical efficacy. ${ }^{81,82}$ Finally, preclinical models suggest that certain chemotherapies and targeted kinase inhibitors induce an immunologic cell death resulting from rapid release of both tumor antigens and self molecules from dying cancer cells, activating toll-like receptor pathways in DCs and promoting inflammation and heightened antitumor immunity. ${ }^{83,84}$ These and many other potentially synergistic treatment combinations are under active exploration and will be required to achieve the true potential of cancer immunotherapy.

\section{AUTHORS' DISCLOSURES OF POTENTIAL CONFLICTS} OF INTEREST

Although all authors completed the disclosure declaration, the following author(s) indicated a financial or other interest that is relevant to the subject matter under consideration in this article. Certain relationships marked with a " $U$ " are those for which no compensation was received; those relationships marked with a " $C$ " were compensated. For a detailed description of the disclosure categories, or for more information about ASCO's conflict of interest policy, please refer to the Author Disclosure Declaration and the Disclosures of Potential Conflicts of Interest section in Information for Contributors.

Employment or Leadership Position: None Consultant or Advisory

Role: Suzanne L. Topalian, Bristol-Myers Squibb (U), Amplimmune (C); George J. Weiner, Genentech (C); Drew M. Pardoll, Bristol-Myers Squibb (U), Amplimmune (C), Aduro Biotech (C), Celgene (C), GlaxoSmithKline (U) Stock Ownership: None Honoraria: Suzanne L. Topalian, Millennium Pharmaceuticals; Drew M. Pardoll, Abbott Laboratories, Immune Design Research Funding: Suzanne L. Topalian, Medarex/Bristol-Myers Squibb; George J. Weiner, Pfizer Expert Testimony: None Other Remuneration: None

\section{AUTHOR CONTRIBUTIONS}

\section{Manuscript writing: All authors \\ Final approval of manuscript: All authors}

\section{REFERENCES}

1. Segal NH, Parsons DW, Peggs KS, et al: Epitope landscape in breast and colorectal cancer. Cancer Res 68:889-892, 2008

2. Schreiber RD, Old LJ, Smyth MJ: Cancer immunoediting: Integrating immunity's roles in cancer suppression and promotion. Science 331:15651570, 2011

3. Disis ML: Immune regulation of cancer. J Clin Oncol 28:4531-4538, 2010

4. McDermott DF, Atkins MB: Application of $\mathrm{IL}-2$ and other cytokines in renal cancer. Expert Opin Biol Ther 4:455-468, 2004

5. Fewkes NM, Mackall CL: Novel gamma-chain cytokines as candidate immune modulators in immune therapies for cancer. Cancer J 16:392-398 2010

6. Weiner GJ: Rituximab: Mechanism of action Semin Hematol 47:115-123, 2010

7. Dall'Ozzo S, Tartas S, Paintaud G, et al: Rituximab-dependent cytotoxicity by natural killer cells: Influence of FCGR3A polymorphism on the concentration-effect relationship. Cancer Res 64: 4664-4669, 2004

8. Weng WK, Levy R: Two immunoglobulin G fragment $C$ receptor polymorphisms independently predict response to rituximab in patients with follicular lymphoma. J Clin Oncol 21:3940-3947, 2003

9. Treon SP, Hansen M, Branagan AR, et al: Polymorphisms in FcgammaRIIIA (CD16) receptor expression are associated with clinical response to rituximab in Waldenström's macroglobulinemia. J Clin Oncol 23:474-481, 2005

10. Clynes RA, Towers TL, Presta LG, et al: Inhibitory $F_{C}$ receptors modulate in vivo cytoxicity against tumor targets. Nat Med 6:443-446, 2000

11. Hudis CA: Trastuzumab: Mechanism of action and use in clinical practice. N Engl J Med 357:39-51, 2007

12. Harbers SO, Crocker A, Catalano G, et al: Antibody-enhanced cross-presentation of self antigen breaks T cell tolerance. J Clin Invest 117:1361 1369, 2007

13. Pawluczkowycz AW, Beurskens FJ, Beum $\mathrm{PV}$, et al: Binding of submaximal $\mathrm{C} 1 \mathrm{q}$ promotes complement-dependent cytotoxicity (CDC) of $B$ cells opsonized with anti-CD20 mAbs ofatumumab
(OFA) or rituximab (RTX): Considerably higher levels of CDC are induced by OFA than by RTX. J Immunol 183:749-758, 2009

14. Wang SY, Racila E, Taylor RP, et al: NK-cell activation and antibody-dependent cellular cytotoxicity induced by rituximab-coated target cells is inhibited by the C3b component of complement. Blood 111:1456-1463, 2008

15. Bowles JA, Wang SY, Link BK, et al: AntiCD20 monoclonal antibody with enhanced affinity for CD16 activates NK cells at lower concentrations and more effectively than rituximab. Blood 108: 2648-2654, 2006

16. Robak T: GA-101, a third-generation, humanized and glyco-engineered anti-CD20 mAb for the treatment of B-cell lymphoid malignancies. Curr Opin Invest Drugs 10:588-596, 2009

17. Nagorsen D, Bargou $R$, Ruttinger $D$, et al: Immunotherapy of lymphoma and leukemia with T-cell engaging BiTE antibody blinatumomab. Leuk Lymphoma 50:886-891, 2009

18. Topp MS, Kufer $P$, Gökbuget $N$, et al: Targeted therapy with the T-cell-engaging antibody blinatumomab of chemotherapy-refractory minimal residual disease in B-lineage acute lymphoblastic leukemia patients results in high response rate and prolonged leukemia-free survival. J Clin Oncol 29: 2493-2498, 2011

19. Younes $A$, Bartlett $N L$, Leonard JP, et al: Brentuximab vedotin (SGN-35) for relapsed CD30positive lymphomas. N Engl J Med 363:1812-1821, 2010

20. Gerber HP, Kung-Sutherland M, Stone I, et al: Potent antitumor activity of the anti-CD19 auristatin antibody drug conjugate hBU12-vcMMAE against rituximab-sensitive and -resistant lymphomas. Blood 113:4352-4361, 2009

21. Loren AW, Porter DL: Donor leukocyte infusions for the treatment of relapsed acute leukemia after allogeneic stem cell transplantation. Bone Marrow Transpl 41:483-493, 2008

22. Chakraverty $R$, Mackinnon S: Allogeneic transplantation for lymphoma. J Clin Oncol 29:18551863, 2011

23. Porter DL, Roth MS, McGarigle $C$, et al.: Induction of graft-versus-host disease as immunotherapy for relapsed chronic myeloid leukemia. N Engl J Med 330:100-106, 1994
24. Mackinnon S, Papadopoulos EB, Carabasi $\mathrm{MH}$, et al: Adoptive immunotherapy evaluating escalating doses of donor leukocytes for relapse of chronic myeloid leukemia after bone marrow transplantation: Separation of graft-versus-leukemia responses from graft-versus-host disease. Blood 86 : 1261-1268, 1995

25. Velardi A: Role of KIRs and KIR ligands in hematopoietic transplantation. Curr Opin Immunol 20:581-587, 2008

26. Topalian SL, Solomon D, Avis FP, et al: Immunotherapy of patients with advanced cancer using tumor-infiltrating lymphocytes and recombinant interleukin-2: A pilot study. J Clin Oncol 6:839-853, 1988

27. Topalian $S L$, Chen $L$, Taube J, et al: Immunology of melanoma, in Balch C, Houghton AN, Sober AJ, et al (eds): Cutaneous Melanoma (ed 5). St Louis, MO, Quality Medical Publishing, 2009, pp 865-882

28. Rosenberg SA, Yannelli JR, Yang JC, et al: Treatment of patients with metastatic melanoma with autologous tumor-infiltrating lymphocytes and interleukin-2. J Natl Cancer Inst 86:1159-1166, 1994

29. Besser MJ, Shapira-Frommer R, Treves AJ, et al: Clinical responses in a phase $\|$ study using adoptive transfer of short-term cultured tumor infiltration lymphocytes in metastatic melanoma patients. Clin Cancer Res 16:2646-2655, 2010

30. Schwartzentruber DJ, Hom SS, Dadmarz R, et al: In vitro predictors of therapeutic response in melanoma patients receiving tumor-infiltrating lymphocytes and interleukin-2. J Clin Oncol 12:14751483, 1994

31. Dudley ME, Yang JC, Sherry R, et al: Adoptive cell therapy for patients with metastatic melanoma: Evaluation of intensive myeloablative chemoradiation preparative regimens. J Clin Oncol 26:52335239, 2008

32. Dudley ME, Wunderlich JR, Yang JC, et al: A phase I study of nonmyeloablative chemotherapy and adoptive transfer of autologous tumor antigenspecific T lymphocytes in patients with metastatic melanoma. J Immunother 25:2434-2251, 2002

33. Yee C, Thompson JA, Byrd D, et al: Adoptive $\mathrm{T}$ cell therapy using antigen-specific $\mathrm{CD} 8+\mathrm{T}$ cell clones for the treatment of patients with metastatic 
melanoma: In vivo persistence, migration, and antitumor effect of transferred T cells. Proc Natl Acad Sci U S A 99:16168-16173, 2002

34. Hunder NN, Wallen $\mathrm{H}$, Cao J, et al: Treatment of metastatic melanoma with autologous $\mathrm{CD} 4+\mathrm{T}$ cells against NY-ESO-1. N Engl J Med 358:26982703, 2008

35. Johnson LA, Morgan RA, Dudley ME, et al: Gene therapy with human and mouse T-cell receptors mediates cancer regression and targets normal tissues expressing the cognate antigen. Blood 114: 535-546, 2009

36. Morgan RA, Dudley ME, Rosenberg SA: Adoptive cell therapy: Genetic modification to redirect effector cell specificity. Cancer J 16:336-341, 2010

37. Robbins PF, Morgan RA, Feldman SA, et al: Tumor regression in patients with metastatic synovial cell sarcoma and melanoma using genetically engineered lymphocytes reactive with NY-ESO-1. J Clin Oncol 29:917-924, 2011

38. Gross G, Waks T, Eshhar Z: Expression of immunoglobulin-T-cell receptor chimeric molecules as functional receptors with antibody-type specificity. Proc Natl Acad Sci U S A 86:10024-10028, 1989

39. Zhong XS, Matsushita M, Plotkin J, et al: Chimeric antigen receptors combining $4-1 \mathrm{BB}$ and CD28 signaling domains augment PI3kinase/AKT/ $\mathrm{BCl}-\mathrm{XL}$ activation and CD8+ T cell-mediated tumor eradication. Mol Ther 18:413-420, 2010

40. Kochenderfer JN, Wilsoh WH, Janik JE, et al: Eradication of B-lineage cells and regression of lymphomna in a patient treate with autologous $T$ cells genetically engineered to recognize CD19. Blood 116:4099-4102, 2010

41. Kohn DB, Dotti G, Brentjens R, et al: CARs on track in the clinic. Mol Ther 19:432-438, 2011

42. Büning $H$, Uckert $W$, Cichutek $K$, et al: Do CARS need a driver's license? Adoptive cell therapy with chimeric antigen receptor-redirected $T$ cells has caused serious adverse events. Human Gene Ther 21:1-4, 2010

43. Zhao $Y$, Moon E, Carpenito $C$, et al: Multiple injections of electroporated autologous $T$ cells expressing a chimeric antigen receptor mediate regression of human disseminated tumor. Cancer Res 70:9053-9061, 2010

44. Pule MA, Savoldo B, Myers GD, et al: Virusspecific $T$ cells engineered to coexpress tumor-specific receptors: Persistence and antitumor activity in individuals with neuroblastoma. Nature Med 14:1264-1270, 2008

45. Pardoll DM, Topalian SL: The role of CD4+ T cell responses in antitumor immunity. Curr Opin Immunol 10:588-594, 1998

46. Rosenberg SA, Yang JC, Restifo NP: Cancer immunotherapy: Moving beyond current vaccines. Nat Med 10:909-915, 2004

47. Kirkwood JM, Ibrahim JG, Sosman JA, et al: High-dose interferon alfa-2b significantly prolongs relapse-free and overall survival compared with the GM2-KLH/OS-21 vaccine in patients with resected stage IIB-III melanoma: Results of intergroup trial E1694/S9512/C509801. J Clin Oncol 19:2370-2380, 2001

48. Kelland L: Discontinued drugs in 2005: Oncology drugs. Expert Opin Investig Drugs 15:13091318, 2006
49. Kantoff PW, Higano CS, Shore ND, et al: Sipuleucel-T immunotherapy for castration-resistant prostate cancer. N Engl J Med 363:411-422, 2010

50. Schwartzentruber DJ, Lawson DH, Richards JM, et al: Gp100 peptide vaccine and interleukin-2 in patients with advanced melanoma. N Engl J Med 364:2119-2127, 2011

51. Hodi FS, O'Day SJ, McDermott DF, et al: Improved survival with ipilimumab in patients with metastatic melanoma. N Engl J Med 363:711-723, 2010

52. Kantoff PW, Schuetz TJ, Blumenstein BA, et al: Overall survival analysis of a phase $I$ randomized controlled trial of a poxviral-based PSA-targeted immunotherapy in metastatic castration-resistant prostate cancer. J Clin Oncol 28:1099-1105, 2010

53. Kenter GG, Welters MJ, Valentijn AR, et al: Vaccination against HPV-16 oncoproteins for vulvar intraepithelial neoplasia. N Engl J Med 361:18381847, 2009

54. Pardoll D: Does the immune system see tumors as foreign or self? Annu Rev Immunol 21 : 807-839, 2003

55. Zou W, Chen L: Inhibitory B7-family molecules in the tumour microenvironment. Nat Rev Immunol 8:467-477, 2008

56. Keir ME, Butte MJ, Freeman GJ, et al: PD-1 and its ligands in tolerance and immunity. Annu Rev Immunol 26:677-704, 2008

57. Pardoll DM: Cancer vaccines. Nat Med 4:525531, 1998

58. Kawai T, Akira S: Toll-like receptors and their crosstalk with other innate receptors in infection and immunity. Immunity 34:637-650, 2011

59. Boon T, Coulie PG, Van den Eynde BJ, et al: Human $T$ cell responses against melanoma. Annu Rev Immunol 24:175-208, 2006

60. Kawakami Y, Rosenberg SA: Human tumor antigens recognized by T-cells. Immunol Res 16 : 313-339, 1997

61. zur Hausen $\mathrm{H}$ : Papillomaviruses in the causation of human cancers: A brief historical account. Virology 384:260-265, 2009

62. Greiner J, Bullinger $L$, Guinn BA, et al: Leukemia-associated antigens are critical for the proliferation of acute myeloid leukemia cells. Clin Cancer Res 14:7161-7166, 2008

63. Thomas AM, Santarsiero LM, Lutz ER, et al: Mesothelin-specific CD8 $+\mathrm{T}$ cell responses provide evidence of in vivo cross-priming by antigenpresenting cells in vaccinated pancreatic cancer patients. J Exp Med 200:297-306, 2004

64. Sharpe AH, Freeman GJ: The B7-CD28 superfamily. Nat Rev Immunol 2:116-126, 2002

65. Leach DR, Krummel MF, Allison JP: Enhancement of antitumor immunity by CTLA-4 blockade. Science: 27:1734-1736, 1996

66. Phan GQ, Yang JC, Sherry RM, et al: Cancer regression and autoimmunity induced by cytotoxic $T$ lymphocyte-associated antigen 4 blockade in patients with metastatic melanoma. Proc Narl Acad Sci USA 100:8372-8377, 2003

67. Yang JC, Hughes M, Kammula U, et al: Ipilimumab (anti-CTLA-4 antibody) causes regression of metastatic renal cell cancer associated with enteritis and hypophysitis. J Immunother 30:825-830, 2007

68. Ribas A: Clinical development of the antiCTLA-4 antibody tremelimumab. Semin Oncol 37: $450-454,2010$
69. Robert $\mathrm{C}$, Thomas $\mathrm{L}$, Bondarenko I, et al: Ipilimumab plus dacarbazine for previously untreated metastatic melanoma. N Engl J Med 364: 2517-2526, 2011

70. Waterhouse $P$, Penninger JM, Timms $E$, et al: Lymphoproliferative disorders with early lethality in mice deficient in CTLA-4. Science 270:985-988, 1995

71. Dong $H$, Strome SE, Salomao DR, et al: Tumor-associated B7-H1 promotes T-cell apoptosis: A potential mechanism of immune evasion. Nat Med 8:793-800, 2002

72. Lipson EF, Taube JM, Chen L, et al: Anti-PD-1 and -B7-H1/PD-L1 monoclonal antibodies, in Gajewski TF, Hodi FS (eds): Targeted Therapeutics in Melanoma. New York, NY, Humana Press, 2011 (in press)

73. Brahmer JR, Drake CG, Wollner I, et al: Phase I study of single-agent anti-programmed death-1 (MDX-1106) in refractory solid tumors: Safety, clinical activity, pharmacodynamics, and immunologic correlates. J Clin Oncol 28:3167-3175, 2010

74. Sznol M, Powderly JD, Smith DC, et al: Safety and antitumor activity of biweekly MDX-1106 (antiPD-1, BMS936558/ONO-4538) in patients with advanced refractory malignancies. J Clin Oncol 28: 205s, 2010 (suppl; abstr 2506)

75. McDermott DF, Drake CG, Sznol M, et al: A phase I study to evaluate safety and antitumor activity of biweekly BMS-936558 (anti-PD-1, MDX1106/ONO-4538) in patients with RCC and other advanced refractory malignancies. J Clin Oncol 29, 2011 (suppl; abstr 331)

76. Lynch DH: The promise of 4-1BB (CD137)mediated immunomodulation and the immunotherapy of cancer. Immunol Rev 222:277-286, 2008

77. Jensen SM, Maston LD, Gough MJ, et al: Signaling through OX40 enhances antitumor immunity. Semin Oncol 37:524-532, 2010

78. Hurwitz AA, Yu TF, Leach DR, et al: CTLA-4 blockade synergizes with tumor-derived granulocytemacrophage colony-stimulating factor for treatment of an experimental mammary carcinoma. Proc Natl Acad Sci U S A 95:10067-10071, 1998

79. Li B, VanRoey M, Wang $C$, et al: Antiprogrammed death-1 synergizes with granulocyte macrophage colony-stimulating factor-secreting tumor cell immunotherapy providing therapeutic benefit to mice with established tumors. Clin Cancer Res 15:1623-1634, 2009

80. Curran MA, Montalvo W, Yagita $H$, et al: PD-1 and CTLA-4 combination blockade expands infiltrating $T$ cells and reduces regulatory $T$ and myeloid cells within B16 melanoma tumors. Proc Natl Acad Sci U S A 107:4275-4280, 2010

81. Okazaki T, Okazaki IM, Wang J, et al: PD-1 and LAG-3 inhibitory co-receptors act synergistically to prevent autoimmunity in mice. J Exp Med 208: 395-407, 2011

82. Humphrey R, Brockway-Lunardi L, Bonk D, et al: Opportunities and challenges in the development of experimental drug combinations for cancer. J Natl Cancer Inst 103:1222-1226, 2011

83. Apetoh L, Ghiringhelli F, Tesniere A, et al: Toll-like receptor 4-dependent contribution of the immune system to anticancer chemotherapy and radiotherapy. Nat Med 13:1050-1059, 2007

84. Ghiringhelli $F$, Apetoh $L$, Tesniere $A$, et al: Activation of the NLRP3 inflammasome in dendritic cells induces IL-1beta-dependent adaptive immunity against tumors. Nat Med 15:1170-1178, 2009 


\section{Glossary Terms}

Adoptive cell transfer (ACT): The culture and expansion of T lymphocytes outside the body and then the infusion of those lymphocytes into patients for therapeutic purposes.

Antibody-dependent cell-mediated cytotoxicity (ADCC): A mechanism of cell-mediated immunity whereby an effector cell of the immune system actively lyses a target cell that has been bound by specific antibodies.

Antigen: A substance that promotes, or is the target of, an immune response.

Antigen-presenting cells (APCs): Cells of the immune system that play a major role in adaptive immunity, APCs are responsible for binding and processing antigens for presentation to T lymphocytes and producing signals that lead to lymphocyte proliferation and differentiation. Dendritic cells and macrophages are examples of APCs.

Biomarker: A functional biochemical or molecular indicator of a biologic or disease process that has predictive, diagnostic, and/or prognostic utility.
Coregulatory receptors: Molecules that transmit additional signals through B-cell and T-cell receptors.

Dendritic cell (DC): The most efficient antigen-presenting cells of the immune system, which play a critical role in the regulation of the adaptive immune response. Immature DCs internalize and process antigens. Their maturation leads to DCs migrating to draining lymph nodes where they prime and activate $\mathrm{T}$ lymphocytes.

Granulocyte macrophage colony-stimulating factor (GM-CSF): A growth factor that stimulates the production of white blood cells. Normally used in cancer therapy and bone marrow transplantation, GM-CSF augments white blood cell production, decreasing the risk of infection. In vaccine therapy, it is an effective vaccine adjuvant administered to activate endogenous dendritic cells, the most effective antigen-presenting cells of the immune system.

Immunogenic: Capable of inducing an immune response.

Immunotherapy: A therapeutic approach that uses cellular and/or humoral elements of the immune system to fight a disease. 